\title{
LA NEUTRALIZACIÓN CULTURAL COMO ESTRATEGIA PARA LA TRADUCCIÓN DE LA AFECTIVIDAD DE MICROCUENTOS
}

\author{
Javiera Ramírez Morales ${ }^{1}$ \\ Mirla Correa Castro ${ }^{1}$ \\ Néstor Singer Contreras ${ }^{1}$ \\ ${ }^{1}$ Universidad de Santiago de Chile, Santiago, Chile
}

\begin{abstract}
Resumen: El objetivo del artículo es describir estrategias para generar un traspaso semántico y afectivo de los aspectos subjetivos presentes en 16 microcuentos del concurso Santiago en 100 palabras. En concreto, este estudio perfila la estrategia de neutralización cultural (Aixelá) como mecanismo para conseguir una equivalencia emocional al traducir dichos textos al inglés. La obtención de los datos se lleva a cabo mediante la implementación de un cuestionario que recopiló las emociones producidas en 32 hablantes de español y en 20 de inglés. A partir de esta información, se ejecutó una metodología de tres etapas: i) propuesta de traducción inicial, ii) validación de traducciones, y iii) edición final de traducciones. Los resultados establecen que la neutralización consigue una equivalencia de emociones en ambas lenguas en un $81 \%$ de los relatos. En el caso de los microcuentos con incongruencia afectiva, se teoriza que esto puede deberse a: i) las propiedades del tipo textual y ii) la subjetividad presente en los lectores. Se concluye que la neutralización cultural es una estrategia apropiada para la traducción de microcuentos al conseguir una equivalencia de emociones significativa del corpus estudiado.
\end{abstract}

Palabras clave: Traducción Literaria; Neutralización Cultural; Emociones

\section{NEUTRALIZATION AS A STRATEGY TO TRANSLATE THE AFFECTIVENESS OF MICROTALES}

Abstract: This article aims to translate the emotional factor in 16 short stories of Santiago en 100 Palabras by exploring new strategies to produce 
a semantic-affective translation. Specifically, this research highlights neutralization (Aixelá) as a means to achieve an emotional equivalence in such text typologies. Data collection was carried out by the implementation of a survey to collect the emotions produced in 32 Spanish speakers and 20 English speakers. From this information, a three-step methodology was performed: i) translation proposal, ii) validation, and iii) final editing. The results indicate emotional equivalence was achieved in $81 \%$ of the short stories. As to those cases of emotional incongruence, it is considered it may be due to three possible reasons: i) textual typology conventions, and ii) emotional subjectivity. In conclusion, cultural neutralization is considered an appropriate strategy to achieve emotional equivalence between these two languages in the selected corpus.

Key words: Literary Translation; Cultural Neutralization; Emotions

\section{Introducción}

La traducción literaria es una de las que más desafíos presenta para poder transmitir las intenciones del autor (Stinson). Lo anterior supone que el traductor, entre sus competencias profesionales, debe ser buen escritor, pues debe preocuparse de que el texto mantenga el mismo estilo y sentido que tiene en la lengua original. A modo de metáfora, Stinson compara el texto literario con una pieza de artesanía, cuyo impacto en los lectores está supeditado a la forma que su artesano le dé. Desde esta perspectiva, la traducción este tipo de textos apunta a imitar tanto la forma como el sentido del texto original en todas sus dimensiones.

Para lograr este efecto, el traductor debe recurrir a una serie de estrategias que le permitan dicho traspaso fidedigno. Trujillo sugiere que, en efecto, las técnicas que el traductor utilice están supeditadas al tipo textual y a la cultura meta. No obstante, existen otros factores que el traductor debe considerar al momento de realizar este tipo de traducciones, en concreto: la intencionalidad emocional o carga emotiva del texto de origen, ya que la literatura es un canal de expresión escrita de las emociones. Por ende, resulta imperioso que dicho elemento sea parte crucial del proceso traductor. 
Los artículos sobre estrategias de traducción literaria (MuñozRico; Quintero and Zaro) parecen privilegiar lo estético por sobre este componente afectivo. No obstante, este estudio pretende establecer si es posible de realizar un traspaso emocional efectivo en microcuentos neutralizando su contenido cultural. En otras palabras, este estudio aborda el traspaso de la carga emocional recurriendo a la técnica de neutralización cultural (Aixelá) en un corpus de 16 microcuentos de Santiago en 100 palabras (Fundación Plagio), concurso que recoge narraciones culturales propias de esta ciudad.

En consecuencia, esta investigación se orienta a dar cuenta sobre los efectos de la neutralización cultural como estrategia de traducción en el mantenimiento de las convenciones textuales del microcuento y en la transmisión de la carga emocional del corpus seleccionado.

a. ¿Hasta qué punto puede la neutralización cultural mantener las convenciones textuales del microcuento?

b. ¿Hasta qué punto es posible transmitir la carga emocional de 16 microcuentos de Santiago en 100 palabras al neutralizar su carga cultural?

\section{Marco teórico}

\subsection{Los desafíos de la traducción literaria}

En general, existe consenso de que la traducción de un texto literario es un desafío, pues en dicho contexto el uso de la lengua tiene finalidades estéticas y efectos de sentido viables de ser alcanzados en el proceso de recepción. Para Jandová, la traducción literaria es una actividad que aparenta funcionar con libertad, mientras que en realidad condiciona y limita la creatividad del traductor, transformando su trabajo en una ilusión. Catenaro señala que la complejidad de la traducción literaria radica en la gran cantidad de responsabilidades que implica esta disciplina, por ejemplo, la 
responsabilidad estética e intelectual del traductor con el texto, y la responsabilidad moral con los lectores de su producción.

Lo anterior supone dos elementos claves para concretar una traducción literaria: la naturaleza del texto a traducir y el destinatario de la traducción. Ambos factores condicionan la traducción hacia distintos enfoques que van a determinar las estrategias que se acogerán al momento de elaborar una traducción. Considerando estos dos elementos como las bases de las dificultades de la traducción literaria, la discusión se sitúa en torno al objeto central de este trabajo, vale decir, la traducción de microcuentos.

\subsection{El microcuento como objeto de crítica}

Piña-Rosales menciona que el microrelato no es el producto de una aleación de tipologías literarias, sino que se constituye como un texto narrativo único en donde predominan aspectos fundamentales como la brevedad, la concisión y la precisión. Cada una de estas características remite a la identificación de tres sistemas claves que actúan de manera reglamentaria en estos relatos. Según Larrea, estos son la brevedad, la transtextualidad y el fragmentarismo. Por un lado, la brevedad es la cualidad empírica más evidente del microcuento. Esta apunta a lo conciso que debe ser un relato, teniendo en cuenta que no existen espacios para que el autor entregue respuestas al lector. Por otro lado, la transtextualidad se refiere a la capacidad de los receptores de comprender el relato más allá de sus propios límites, donde también entra en juego la intertextualidad, la que se vincula a la construcción de relaciones entre el texto que se lee con otros textos. Finalmente, el fragmentarismo se entiende como una condición que segmenta el relato y que se relaciona derechamente con la brevedad, ya que la reducida extensión del microcuento genera rupturas en la lectura y exige un complejo proceso de realización de inferencias.

En consecuencia, un microcuento constituye una organización abierta compleja, cuya extensión reducida alberga una serie de elementos culturales necesarios para su interpretación. Por lo 
tanto, si se extrapola este tipo de texto a la traducción, dichas características se perfilarían como las principales dificultades al momento de traducir.

\subsection{La traducción de microcuentos}

Los microcuentos, al ser un género rupturista y relativamente reciente (Zavala), son un reto para los autores, lectores y, desde luego, traductores. En base a esta última disciplina, Marks apunta a que gran parte de lo que convierte a la traducción de microcuentos en una actividad tan desafiante es esa dificultad para determinar cómo se puede conseguir una traducción de microcuentos notable y excepcional, pues así como las posibilidades de interpretación de estos relatos son infinitas, también existen un gran número de alternativas de traducción.

Por su parte, Newmark asegura que una de las mayores complicaciones de la traducción de microcuentos es su brevedad, ya que en este tipo de relatos, el traductor debe estar siempre muy consciente de las palabras que va a utilizar y de su significado; es crucial que se transmita con precisión el contenido del texto en un formato que comúnmente no supera las 100 palabras. Además, destaca que otro de los grandes desafíos del traductor de microcuentos es el factor cultural dado que generalmente se abordan temáticas cotidianas de la vida de un grupo de personas o de una cultura. Desde la posición del lector, los microcuentos demandan cierta familiaridad con la cultura y el entorno que rodea al relato. En el caso del traductor, la situación es más compleja, pues debe estar familiarizado con la cultura hacia la que está traduciendo para primeramente entender el contexto en el que se desarrolla la historia y, posteriormente, transmitir la intención del autor. No obstante, el desafío yace en cómo traspasar toda la carga cultural de un microcuento si las culturas en juego no comparten aspectos similares, considerando las características estilísticas propias del formato. Como respuesta, Marks propone que la mejor alternativa es dejar la interpretación en las manos de los lectores 
de la traducción y esperar a que estos estén lo suficientemente familiarizados con la historia para que comprendan el relato como una única pieza y no como fragmentos independientes.

Sin embargo, este trabajo considera la capacidad del traductor como base para ponderar los principales componentes del microcuento y, posteriormente, adoptar estrategias que apunten a mantener los elementos propios de esta tipología y, como fin clave, lograr equivalencias en una dimensión clave del microcuento: las emociones producidas.

\subsection{La traducción de la dimensión afectiva de un microcuento}

La lectura de un microcuento puede resultar en diferentes reacciones afectivas. Para Raymond et al., las emociones son piezas fundamentales para la construcción de una experiencia lectora, ya que lo que el lector espera de un microcuento, o incluso de una novela, es que la historia lo estremezca emocionalmente.

Respecto a la traducción de dichas emociones, Henitiuk asegura que al percibir, regular y transferir emociones mediante un medio escrito, el traductor conforma un texto que contiene información emocional, que incluye las posibles reacciones de los lectores meta y que da cabida a nuevas interpretaciones e intertextualidades.

En cuanto a una posible categorización de estas emociones, Vigotsky propone que estas se pueden clasificar como primarias y secundarias. Las emociones primarias son aquellas emociones innatas que suelen ir acompañadas de claras e inmediatas respuestas físicas y fisiológicas, como el llanto que produce la tristeza, y la risa, que produce la alegría. Por su parte, las emociones secundarias son aquellas que resultan de la combinación de las emociones primarias y como producto de las experiencias del individuo en un determinado contexto, tal como la vergüenza o la culpa. Por sobre esta categorización, a fin de especificar aún más cada emoción, se acoge también una subdivisión de emociones positivas y negativas (Smith and Lazarus) en relación con la experiencia lectora de cada individuo. 
En vista de lo anterior, dentro de este estudio, las emociones son el objetivo principal de los microcuentos $\mathrm{y}$, a fin de facilitar la transferencia y la mantención de estas, se estima que mediante la neutralización cultural de los principales referentes culturales de estos microcuentos es posible generar las mismas reacciones emocionales en la lengua meta a pesar de las limitaciones propias del tipo textual.

\subsection{Neutralización de aspectos culturales}

A la fecha de redacción de este artículo, la idea de neutralizar el factor cultural para preservar la carga emocional de un microcuento no se considera como una opción dentro de las distintas estrategias de traducción literaria para el traspaso de emociones (Martínez; Quintero and Zaro; Revuelta). No obstante, sería relevante evaluar empíricamente la pertinencia de una estrategia como esta en tipos textuales cargados de unidades culturales, como los microcuentos. Esta estrategia mantiene el contenido afectivo del relato mientras se generalizan los elementos propios de una cultura. En este sentido, la neutralización no debe interpretarse como una pérdida de elementos o convenciones del tipo textual, sino como medio para poder generar la misma emoción en la lengua meta, que se concibe como fin último de la traducción del microcuento.

En concreto, la neutralización es una técnica de traducción que se basa en sustituir el referente cultural de la lengua origen por otra elección léxica que sea más neutra y que esté libre de marcas culturales (Aixelá). Su utilización es objeto de controversia y más aún cuando se emplea en un contexto literario. En general, esta estrategia se percibe como la opción más sencilla para el traductor cuando debe lidiar con situaciones complejas de traducir. Se cree que el traductor neutralizó su traducción al no encontrar otra técnica para utilizar, como: calcos, préstamos, creaciones léxicas, explicitación y adaptación cultural (Martínez). No obstante, esta técnica tiene el potencial de actuar como un nexo cultural. En este sentido, este estudio pretende ser un primer acercamiento hacia la 
validación de la neutralización como estrategia para la traducción de microcuentos, enfatizando la carga afectiva y resultado emocional en la lengua meta.

\section{Metodología}

La investigación corresponde a un estudio exploratoriodescriptivo enmarcado dentro de la traducción de microcuentos y traspaso de su carga afectiva. Para esto, se diseñó un cuestionario para recabar las emociones generadas a partir de los 16 microcuentos ganadores del concurso Santiago en 100 palabras para dos grupos no-aleatorios e intencionados. Estos grupos de participantes corresponden un grupo de 32 alumnos chilenos de la carrera de Lingüística Aplicada a la Traducción de la Universidad de Santiago de Chile (USACH) y 20 alumnos norteamericanos del Instituto International Honors Program (IHP por sus siglas en inglés). En el caso del grupo chileno, los participantes eran 1) hablantes nativos de español, 2) menores de 20 años cursando su primer año en la carrera, 3) con residencia mínima de 5 años en la ciudad de Santiago, y 4) no presentaban impedimentos visuales serios. Estos criterios apuntaban a generar una muestra que tuviera conocimiento de la lengua en sus particularidades locales, así como conocimiento las convenciones culturales de la ciudad. Al ser alumnos de primer año, su competencia lingüística les permitía leer de manera competente el texto, pero sin tener los conocimientos adicionales para analizarlos en términos de estructura, estética, etc. Por su parte, los participantes del grupo norteamericano cumplían con requisitos similares: ser hablantes nativos del inglés, tener menos de 20 años y no presentar problemas visuales importantes que pudieran interferir con su comprensión lectora.

El cuestionario consistía en una lectura de cada microcuento, donde cada alumno debía responder con sustantivos de emociones que se desprendían de su lectura con un máximo de 4 por cuento. En el caso del cuestionario en español, se utilizaron los microcuentos 
tal como aparecen en sus versiones finales publicadas, mientras que las traducciones utilizadas en la versión en inglés del cuestionario fueron elaboradas en tres etapas: traducción inicial utilizando la neutralización cultural, validación de las traducciones por parte de un grupo similar a la muestra utilizada en inglés y postedición de las traducciones. La neutralización fue utilizada en casos que la carga cultural unidades de traducción: 1) tuviesen más de una alternativa de traducción, 2) representasen una complejidad semántica o sintáctica, o 3) presentasen una dificultad en su proceso de neutralización. Cabe señalar que se consideraron las emociones más significativas encontradas por el grupo de habla española como punto de partida para realizar las traducciones al inglés. Las versiones finales de los cuestionarios en ambas versiones fueron validadas por 5 expertos en traducción y especialistas en inglés como lengua extranjera.

El cuestionario fue aplicado primero al grupo hispanohablante y posteriormente al grupo norteamericano. En ambos casos, los participantes dispusieron de 20 minutos para responder. Los autores llevaron a cabo esta aplicación en sesiones dentro del horario de clases de los participantes a la misma hora para evitar posibles fuentes de invalidación interna en la recolección de datos.

Posteriormente, se analizaron y agruparon las reacciones afectivas resultantes en grupos del mismo tipo mediante la creación de macrocategorías según el modelo propuesto por Vigotsky, vale decir, primarias y secundarias. Aquellas emociones que no representaban una proporción dominante dentro del universo de respuestas se clasificaron en una tercera macrocategoría denominada misceláneas. Dentro de las macrocategorías primarias y secundarias, se crearon subcategorías para determinar la carga predominante en cada grupo: positivas y negativas. Para establecer las cargas predominantes en las narraciones, se aplicó una asociación de conceptos para amalgamar los marcos y subcategorías obtenidos. De esta manera, la emoción con mayor frecuencia según la evaluación de los lectores hispanohablantes se consideró como referente y se complementó con todas aquellas 
que compartían la misma carga. Por ejemplo, en el cuento $\mathrm{N}^{\circ} 1$, la emoción predominante identificada fue la tristeza y, bajo ella, se agruparon otras emociones como la pena, nostalgia, melancolía y angustia. En consecuencia, se establece que el porcentaje mínimo para que la emoción sea representativa es de $50 \%$.

Este estudio se llevó a cabo según las normas del Comité de Ética de la Universidad de Santiago de Chile. En el caso de este estudio, los alumnos firmaron un consentimiento informado donde se detallaban sus derechos en cuanto a la anonimidad de su participación, así como el uso de la información generada para redacción de una tesis y posibles publicaciones adicionales que resultasen de dicho trabajo.

\section{Resultados}

En relación con el corpus, 15 cuentos fueron traducidos en 100 palabras o menos, mientras que solo uno tuvo un margen de $10 \%$ por sobre el máximo del concurso. De esta manera, se puede establecer que las propuestas de traducción sí cumplían con las características estilísticas propias del microcuento, o sea, su extensión. Por otra parte, se puede establecer que las emociones expresadas por los encuestados de ambos grupos son prácticamente las mismas. Cuantitativamente hablando, de un total de 16 microcuentos, el $81 \%$ equivale a los relatos en los que sí se logró un traspaso afectivo satisfactorio mediante la neutralización cultural, esto es, 13 microcuentos. Por otra parte, el 19\% restante representa aquellos relatos en los que no se alcanzó el objetivo, vale decir, 3 microcuentos. La Figura 1 muestra los resultados generales, mientras que la Tabla 1 muestra la correspondencia o no correspondencia de emociones en los 16 microcuentos. 


\section{Traspaso afectivo exitoso No equiwalencia afectiva}

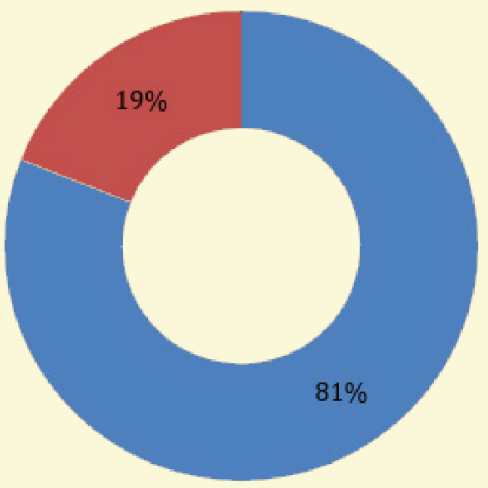

Figura 1. Evaluación comparativa de los resultados obtenidos.

\begin{tabular}{|c|c|c|c|c|c|c|}
\hline \multirow{2}{*}{ Cuentos } & \multicolumn{2}{|c|}{ Español } & \multicolumn{2}{c|}{ Inglés } \\
\cline { 2 - 7 } & Categoria & Emocion relevante & Porcentaje & Categoria & Emocion relevante & Porcentaje \\
\hline Cuento $N^{\circ} 1$ & Primaria & tristeza & $81 \%$ & Primaria & tristeza & $92 \%$ \\
\hline Cuento $N^{\circ} 2$ & Secundaria & ansiedad & $95 \%$ & Primaria & tristeza & $78 \%$ \\
\hline Cuento $N^{\circ} 3$ & Primaria & tristeza & $69 \%$ & Primaria & miedo & $81 \%$ \\
\hline Cuento $N^{\circ} 4$ & Secundaria & confusión & $82 \%$ & Primaria & alegría & $87 \%$ \\
\hline Cuento $N^{\circ} 5$ & Primaria & rabia & $69 \%$ & Primaria & rabia & $69 \%$ \\
\hline Cuento $N^{\circ} 6$ & Secundaria & nostalgia & $66 \%$ & Primaria & tristeza & $86 \%$ \\
\hline Cuento $N^{\circ} 7$ & Primaria & alegría & $84 \%$ & Primaria & alegría & $82 \%$ \\
\hline Cuento $N^{\circ} 8$ & Secundaria & soledad & $85 \%$ & Primaria & tristeza & $99 \%$ \\
\hline Cuento $N^{\circ} 9$ & Primaria & alegría & $95 \%$ & Primaria & alegría & $81 \%$ \\
\hline Cuento $N^{\circ} 10$ & Secundaria & indiferencia & $77 \%$ & Primaria & felicidad & $95 \%$ \\
\hline Cuento $N^{\circ} 11$ & Primaria & alegría & $92 \%$ & Primaria & tristeza & $82 \%$ \\
\hline Cuento $N^{\circ} 12$ & Primaria & alegría & $91 \%$ & Primaria & amor & $96 \%$ \\
\hline Cuento $N^{\circ} 13$ & Primaria & alegría & $91 \%$ & Primaria & felicidad & $87 \%$ \\
\hline Cuento $N^{\circ} 14$ & Primaria & tristeza & $58 \%$ & Primaria & tristeza & $79 \%$ \\
\hline Cuento $N^{\circ} 15$ & Primaria & tristeza & $84 \%$ & Primaria & tristeza & $90 \%$ \\
\hline Cuento $N^{\circ} 16$ & Secundaria & curiosidad & $73 \%$ & Secundaria & intriga & $68 \%$
\end{tabular}

Tabla 1. Correspondencia o no correspondencia de emociones.

En el siguiente apartado se problematizarán aquellos cuentos en los que no ocurrió una equivalencia. En concreto, se analizarán aquellos casos en los que las interpretaciones de un microcuento 
generaron percepciones opuestas, como ocurrió con el cuento Selva negra en la Tabla 2.

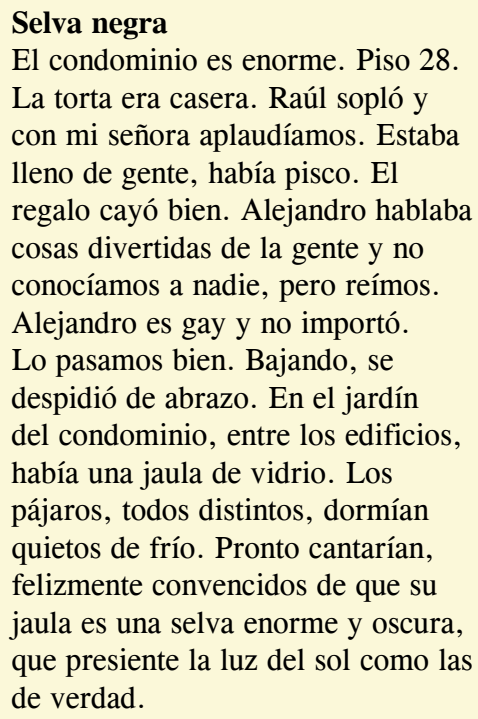

\section{Dark jungle}

The condo is huge. 28th floor. The cake was homemade. Raul blew out the candles and my wife and I clapped. The place was crowded, there was alcohol. The present was appreciated. Alejandro was making fun of people and we didn't know anyone but we still laughed. He is gay but it didn't matter. We had fun. When leaving, Alejandro hugged us. In the condo's garden, between the buildings, there was a glass cage. The birds, all different, were sleeping frozen stiff. They would soon sing, happily convinced that their cage is an immense dark jungle, which feels the sunlight like the real ones.

Tabla 2. Selva negra (2004) en su versión en español e inglés.

En este cuento, los encuestados del grupo en español manifestaron que la emoción predominante en el relato era la confusión con un $82 \%$, mientras que el grupo en inglés destacó la alegría con un $87 \%$. Se cree que esto podría ser producto de dos factores: el fragmentarismo de la tipología textual y la capacidad de interpretar la analogía del relato. En general, este microcuento presenta una estructuración clara y, estilísticamente, es cohesivo. Sin embargo, el fragmentarismo característico de estos relatos genera una confusión entre las tramas que envuelve el texto, pues Selva negra narra dos situaciones conectadas semánticamente mediante una analogía. Por un lado, el cuento comienza describiendo a un grupo de personas en un ambiente de festejo y, por otro, se retrata un contexto oscuro dentro de una jaula de pájaros. A partir de 
esta interacción, la alegría que provoca la celebración del inicio se tensiona con la alusión a una situación de opresión no percibida que genera la referencia a los pájaros. De esta manera, se considera que los encuestados de los dos instrumentos enfocaron su interés en diferentes partes del relato, o bien, emplearon habilidades diversas para establecer los nexos simbólicos entre las dos situaciones representadas.

Nadan (ver Tabla 3.) es otros de los microcuentos con falta de equivalencia en las respuestas afectivas.

\begin{tabular}{l}
\hline Nadan \\
Se encuentran todos los lunes. Nunca \\
se saludan en la superficie. Son \\
imágenes difusas las que tienen el uno \\
del otro porque el agua les empaña \\
los lentes. Al principio nadan muy \\
rápido, con ansiedad. Luego lo hacen \\
al mismo tiempo, más pausadamente, \\
como ahogándose y riéndose a la \\
vez. Ella sale primero de la piscina. \\
Se tapa con la toalla apenas sube la \\
escalera metálica. Él espera algunos \\
minutos. Flotando boca arriba, mira \\
las nubes a través del techo de vidrio. \\
En sus camarines se duchan cantando \\
para sacarse el olor a cloro que les \\
queda en la piel.
\end{tabular}

\section{Nadan}

se saludan en la superficie. Son imágenes difusas las que tienen el uno del otro porque el agua les empaña los lentes. Al principio nadan muy rápido, con ansiedad. Luego lo hacen como ahogándose y riéndose a la vez. Ella sale primero de la piscina. Se tapa con la toalla apenas sube la escalera metálica. Él espera algunos minutos. Flotando boca arriba, mira En sus camarines se duchan cantando para sacarse el olor a cloro que les queda en la piel.

\section{They just swim}

They meet every Monday. They never say hello on the surface. They have a blurry picture of each other because water clouds their goggles. Initially, they swim rapidly and eagerly.

Then they do it at the same time, slowly, as if they were drowning and laughing all together. She gets out of the pool first. She covers her body with a towel as soon as she climbs up the metallic steps. He waits a few minutes, floating face up and looking at the clouds through the glass roof. In their dressing rooms they sing while they take a shower to remove the smell of chlorine covering their skin.

Tabla 3. Nadan (2010) en su versión en español e inglés.

Los encuestados del grupo en español señalaron que la indiferencia dominaba por sobre otras emociones con un $77 \%$, mientras que el grupo en inglés indicaron que era la felicidad con un $95 \%$. Ante esta evidente diferencia de emociones, se estima que podría ser consecuencia del estilo narrativo del autor. En particular, se identifica que la redacción del relato no despierta muchos instintos afectivos en los lectores en español dado que 
la naturaleza de la narración no presenta cambios de matices en la trama. Por este motivo, es posible que los encuestados no reconocieran una emoción definida en el relato. Además, puede incidir la capacidad de comprensión de un texto complejo o pocos recursos lingüísticos para precisar la emoción subyacente. Esto se desprende de la indiferencia provocada por el cuento, pues entre las respuestas recopiladas se repitió cuatro veces el sustantivo "nada", quizás a modo de asociación con el título. En base a esto, es posible que se trate de una estrategia del autor para redactar la historia de una manera imperceptible, sin apuntar demasiado a la memoria emotiva de sus lectores, en función de ejemplificar y reproducir el contexto general de la trama.

El grupo en inglés, en cambio, sí entregó una connotación a la lectura. Frente a esto, se analiza el alcance de la traducción del relato. Es importante mencionar que en este texto no se aplicó la estrategia de neutralización cultural debido a que no contenía culturemas. En consecuencia, se presume que la forma de la propia anatomía de este tipo textual se interpone en la traducción. En concreto, el título de la historia potencialmente construye la interpretación y entrega sentido crítico a los lectores. De esta manera, es posible que los encuestados al no conocer las intenciones detrás de Nadan, (traducido como They just swim) solo se enfocaron en analizar la trama del microcuento y en base a eso determinaron la emoción dominante.

Finalmente, el contraste de emociones más significativo ocurrió en el cuento Soldado de terracota, detallado en la Tabla 4.

\section{Soldado de terracota}

Li Piang Hua, soldado de terracota del emperador Qin Shi Huang, escapó de los subterráneos de La Moneda. Caminó hacia el norte, pasó la noche tomando chelas con unos emigrantes peruanos. Conoció a una chica de Rengo que vino a

\section{The terracotta warrior}

Li Piang Hua, a terracotta warrior of the Emperor Qin Shi Huang, escaped from the government palace museum. He walked northwards and spent the night drinking cold ones with some Peruvian immigrants. He met a woman from a small town who came 


\begin{tabular}{|c|c|}
\hline $\begin{array}{l}\text { Santiago persiguiendo una hora } \\
\text { plan AUGE para un oftalmólogo. } \\
\text { Ahora viven en La Pintana y tienen } \\
\text { un puesto de comida china. Es } \\
\text { noche. En un pequeño patio junto } \\
\text { a un triciclo y dos balones de gas, } \\
\text { Li ejecuta una silenciosa danza; } \\
\text { blande su ballesta apuntando a la } \\
\text { luna. En sus oídos, canto de bambú } \\
\text { acariciado por viento. }\end{array}$ & $\begin{array}{l}\text { here looking for an ophthalmologist } \\
\text { appointment through a healthcare } \\
\text { program. Now, they live in the slums } \\
\text { and have a Chinese food stall. At } \\
\text { night, in a small yard next to a tricycle } \\
\text { and two gas cylinders, Li performs } \\
\text { a noiseless dance. He wields his } \\
\text { crossbow pointing to the moon, while } \\
\text { in his ears; the sound of bamboo is } \\
\text { caressed by wind. }\end{array}$ \\
\hline
\end{tabular}

Tabla 4. Soldado de terracota (2011) en su versión en español e inglés.

Los encuestados del grupo en español señalaron que la emoción más representativa del relato era la alegría con un $92 \%$. Mientras que el grupo en inglés resaltó la tristeza con un $82 \%$. Ante esta dualidad de emociones contradictorias, se identifica un solo posible factor: la neutralización de los referentes culturales del relato. En el caso del texto original, se reconoce a estos elementos identitarios como "distractores" de la atención del lector, en otras palabras, se perciben como unidades que sobresalen del resto del texto. Estas llegan incluso a permanecer en la memoria del receptor como el único mensaje significativo de toda la lectura, moviendo el foco de la trama de la narración. Por esta razón, se presume que los encuestados establecen un grado tal de proximidad con el microcuento, que les permite apreciar y valorar ciertos aspectos de su cultura desde la percepción de espectador. En consecuencia, aquellas situaciones íntimas de un colectivo en particular se transforman en contenido accesible y familiar que resulta en una reacción afectiva de alegría.

No obstante, en el grupo en inglés, la implementación de la estrategia de neutralización cambió radicalmente el foco interpretativo de los lectores del grupo. Esto porque, al haberse neutralizado el componente cultural del microcuento, los encuestados distinguieron el trasfondo del relato, antes de cualquier otro elemento del texto, vale decir, la tristeza del protagonista que emigra de su hogar para asentarse en otro país. A partir de esta 
diferencia en la percepción del texto, se hace necesario destacar que el estado neutro de los referentes culturales permitió que una de las principales cualidades del tipo de texto predominará en la lectura del microcuento: la intertextualidad. La emoción resultante en este grupo, puede vincularse a la manera en que los encuestados se vieron reflejados en el personaje de la historia, de alguna manera, la realidad de cada lector se combina con la realidad retratada del microcuento y acaba en una reacción afectiva dominante.

En este cuento en particular, es posible conocer el valor de los aspectos culturales presentes en un relato y cómo estos mismos funcionan al ser neutralizados en una traducción. En relación a la discrepancia en los resultados obtenidos, se puede evidenciar que el factor cultural ejerce una gran carga en la interpretación de un relato, dado que sobresale independientemente del texto por la manera creativa de representar nuestra cultura y, a veces, llega incluso a desviar la reacción afectiva de los lectores respecto a la trama desarrollada. Sin embargo, existe un punto relevante dentro de esta discusión, ya que la presencia de referentes culturales en parte de la serie de microcuentos traducidos no siempre generó incongruencia de emociones. Por ejemplo, el relato El Jony, la Dámaris y la Tais (2012) fue identificado como uno de los cuentos con un alto porcentaje de unidades de índole cultural; no obstante, esta situación no derivó en una diferencia de emociones bajo la percepción de ambos grupos objetivos. En consecuencia, se estima que esta incongruencia puede ser el uso de culturemas propios de subculturas, lo que hace aún más complejo el proceso traductor para lograr la equivalencia emocional.

En vista de estos resultados, se enfatizan tres elementos clave que incidieron en la declaración afectiva de cada uno de los encuestados de este proyecto: 1) las características propias del tipo textual; 2) la condición subjetiva del plano emocional y 3) la neutralización de los referentes culturales. Adicionalmente, se establece que el uso de la neutralización cultural no impactó en las convenciones propias de la tipología del microcuento. 


\section{Conclusiones}

El presente estudio corresponde a la primera investigación sistemática que evalúa el uso de la neutralización cultural como estrategia para la traducción de microcuentos chilenos. A través de esta técnica de traducción, se logró un traspaso del plano semántico y gramatical conservando las convenciones propias de este tipo textual así como un traspaso afectivo. Si bien los resultados permiten establecer que en este corpus la neutralización cultural operó efectivamente en el traspaso de la carga emotiva, también se obtuvo discrepancias en tres de los cuentos traducidos.

En primer lugar, se debe clarificar que el objeto mismo de estudio de este trabajo, vale decir, la carga afectiva o emociones que se desprenden de los textos, constituye en sí mismo una materia de carácter subjetivo. En consecuencia, si bien es posible establecer tendencias generalizadas de reacciones afectivas, es vital señalar que cada una de estas interpretaciones corresponde a una reacción individual de cada sujeto, por lo que el factor individual resulta crucial para una mayor comprensión de la incongruencia de la emoción entre ambas lenguas.

El segundo punto se relaciona con las características narrativas y estructurales del microcuento. La brevedad y el fragmentarismo se presentan como elementos que pueden intervenir en la reconstrucción emocional del cuento en la lengua meta, pues dichas características de la anatomía del microcuento afectan la representación cognitiva y afectiva de los encuestados, como por ejemplo, el rol de los títulos en la generación de la emoción meta. En este caso, la propuesta de traducción puede guiar la interpretación emotiva de la narración. Por otra parte, es importante considerar el proceso de comprensión lectora por parte de los participantes, pues esto también puede facilitar en la interpretación del microcuento.

Por último, la neutralización de los referentes culturales puede, efectivamente, incidir en la interpretación afectiva del cuento. De los resultados obtenidos, se evidenció que en aquellos microcuentos con un alto porcentaje de culturemas, la neutralización orientó al 
lector hacia una simplificación de la trama del relato por sobre otros elementos del texto en la lengua meta, mientras que en el texto original la presencia de un alto número de elementos culturales podría haber dificultado la comprensión de la trama, o bien, dirigido la atención hacia los propios referentes culturales.

Por estas razones, se considera que este trabajo podría potencialmente explorar una nueva línea de investigación respecto al empleo de la neutralización cultural en traducción literaria, pues tras los resultados obtenidos en este estudio evidencian la pertinencia de la neutralización entre las estrategias traductológicas en textos de literatura, especialmente cuando se trabaja la dimensión afectiva de los microcuentos.

\section{Referencias}

Aixelá, Javier Franco. "Culture-specific ítems in Translation." En: Álvarez, Roman y Vidal, Carmen-África (Ed). Translation, Power, Subversion. Clevedon: Multilingual Matters, 1996, p. 52-78.

Canterano, Bárbara. "La obra literaria: posibilidades y límites del traductor." Espéculo: Revista de estudios literarios, 2008, s.p.

Fundación Plagio. "Ganadores." Santiago en 100 Palabras. Fundación Plagio. 2018. < http://www.santiagoen100palabras.cl> .

Henitiuk, Valerie. "Literary translation as a reflective process." En: Wilson, Rita y Gerber, Leah (Ed). Creative constraints: Translation and Authorship. Clayton: Monash University Publishing, 2012, p. 3-21.

Jandová, Jarmila. "La creatividad del traductor literario y la ilusión de traducción." Literatura: teoría, historia, crítica 19.2 (2017): 291-314.

Cad. Trad., Florianópolis, v. 39, no 3, p. 189-208, set-dez, 2019. 
Larrea, María Isabel. "Estrategias lectoras en el microcuento." Estudios filológicos n. 39 (2004): 179-190.

Martínez, Cristina. La traducción de los referentes culturales. El caso de ocho apellidos vasco (español-inglés). Tesis. Universitat Jaume I, 2015.

Marks, Cynthia. Translating a Short Story by Hans-Jürgen Greif: Cats, Opera and Proverbs. Tesis. University of Alberta, 2012.

Muñoz-Rico, Ítalo. Las técnicas de traducción y las figuras literarias en la traducción al español de Mémoires d'Hadrien*. Lenguaje 35.2 (2007): 167-196.

Newmark, Peter. Paragraphs on Translation. Clevedon: Multilingual Matters, 1993.

Piña-Rosales, Gerardo. "El cuento: Anatomía de un género literario." Hispania 92.3 (2009): 479-482.

Quintero, Marianella, y Zaro, Juan Jesús. "Problemas y estrategias de traducción en el cambio de código en la literatura chicana al español. El caso de From this wicked patch of dust de Sergio Troncoso.” Núcleo, no. 31, 2014, p. 247-273.

Raymond, Mar A.; Oatley, Keith; Djikic, Maja, y Mullin, Justin. "Emotion and narrative fiction: Interactive influences before, during, and after reading." Cognition \& Emotion 25.5 (2011): 818-833.

Revuelta, Laura. "Estrategias de domesticación y extranjerización en la traducción literaria: Análisis comparativo de dos traducciones al castellano de la novela británica Arabella (1949) de Georgette Heyer." Tesis. Universidad Pontifica de Comillas, 2017.

Smith, Craig, y Lazarus, Richard. "Appraisal components, core relational themes, and the emotions." Cognition and Emotion 7.3-4 (1990): 233-269.

Stinson, Mirta. "La traducción literaria como proceso." En: Badenes, Guillermo y Coisson, Josefina (Ed). Traducción Periodística y Literaria. Córdoba: Comunicarte, 2007. 
Trujillo, Verónica. "La adaptación como técnica de traducción. Taxonomía y estudio de sus funciones aplicada a la traducción lexicográfica." Revue Roumaine de Linguistique 54.4 (2014): 351-367.

Vigotsky, Lev. Teoría de las emociones. Madrid: Akal, 2004.

Zavala, Lauro. "De la teoría literaria a la minificción posmoderna." Ciencias Sociais Unisinos, 43.1 (2007): 86-96.

Recebido em: 20/03/2019

Aceito em: 10/07/2019

Publicado em: Setembro de 2019

Javiera Ramírez Morales E-mail: ramirezjavieram@gmail.com.

ORCID: https://orcid.org/0000-0002-0372-9345

Mirla Correa Castro. E-mail: correacastromirla@gmail.com.

ORCID: https://orcid.org/0000-0002-4136-0120

Néstor Singer Contreras. E-mail: nestor.singer@usach.cl.

ORCID: http://orcid.org/0000-0001-7772-9906 